\title{
An Omnidirectional MEMS Ultrasonic Energy Harvester for Implanted Devices
}

\author{
Anthony G. Fowler, Student Member, IEEE, S. O. R. Moheimani, Fellow, IEEE, \\ and Sam Behrens, Member, IEEE
}

\begin{abstract}
This paper presents the design and characterization of a microelectromechanical systems (MEMS)-based energy harvester with target applications, including implanted biomedical sensors and actuators. The harvester is designed to utilize ultrasonic waves from an external transmitter for mechanical excitation, with electrostatic transducers being used to convert the vibrations of a central mass structure into electrical energy. The device features a novel 3-degrees of freedom design, which enables energy to be produced by the harvester in any orientation. The harvester is fabricated using a conventional siliconon-insulator MEMS process, with experimental testing showing that the system is able to generate $24.7,19.8$, and $14.5 \mathrm{nW}$ of electrical power, respectively, via the device's $x$-, $y$-, and $z$-axis resonance modes over a 15 -s period.

[2013-0293]
\end{abstract}

Index Terms-Energy harvesting, energy scavenging, electrostatic transducer, implantable biomedical devices.

\section{INTRODUCTION}

$\mathbf{T}$ HE RUNNING time of standalone electronic systems such as wireless network nodes, personal electronic devices, and remote sensors is typically dependent on the capacity of the batteries used to power the system [1]-[3]. For this reason there has been much interest in the concept of energy harvesting, where energy from an external source is converted into electrical energy and used to help power the system. This leads to an increase in the effective running time of the device and may even allow the system to become fully self-powered, negating the need for additional external charging.

Energy harvesting has been a particularly relevant application for microelectromechanical systems (MEMS). The ease of integration of MEMS-based devices with external electronic systems, together with their relatively straightforward fabrication process, make them especially suited to the

Manuscript received September 17, 2013; revised March 12, 2014; accepted March 29, 2014. Date of publication May 5, 2014; date of current version November 25, 2014. This work was supported in part by the Australian Research Council; in part by the Commonwealth Scientific and Industrial Research Organisation; and in part by the University of Newcastle, Callaghan, Australia. Subject Editor X. Wang.

A. G. Fowler and S. O. R. Moheimani are with the School of Electrical Engineering and Computer Science, University of Newcastle, Callaghan, NSW 2308, Australia (e-mail: anthony.fowler@uon.edu.au; reza.moheimani@newcastle.edu.au).

S. Behrens is with the Commonwealth Scientific and Industrial Research Organisation Energy Technology, Newcastle upon Tyne, NSW 2300, Australia (e-mail: sam.behrens@csiro.au).

This paper has supplementary downloadable multimedia material available at http://ieeexplore.ieee.org provided by the authors. This includes three .mp4 files animating a fabricated MEMS energy harvester's X-, Y-, and Z-axis' resonance modes, experimentally measured using a 3-D Micro System Analyzer. This material is $413 \mathrm{kB}, 392 \mathrm{kB}$, and $383 \mathrm{kB}$ in size, respectively.

Color versions of one or more of the figures in this paper are available online at http://ieeexplore.ieee.org.

Digital Object Identifier 10.1109/JMEMS.2014.2315199

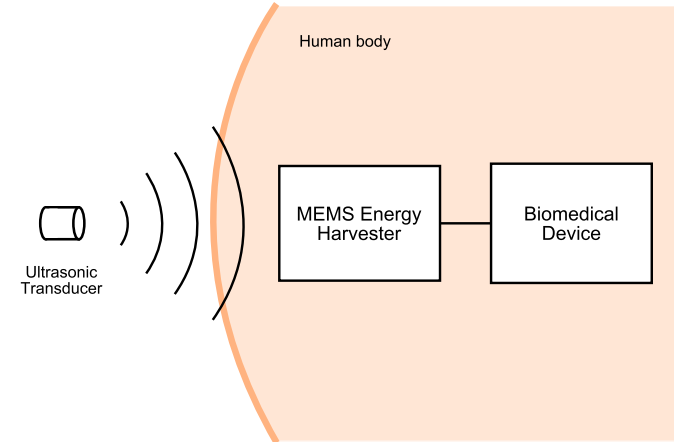

Fig. 1. Ultrasonic energy harvesting concept.

harvesting of energy for microscale applications such as structure monitoring systems and wearable electronics [4], [5]. Such MEMS-based energy harvesters are typically designed to generate electrical energy from vibration sources present within the system's environment [6]-[9].

Implanted electronic devices for biomedical applications such as patient monitoring and drug delivery provide an example where the benefits of microscale energy harvesting are particularly applicable [10]. Such devices continue to use batteries to meet their energy requirements, with surgical procedures being required to replace the batteries when they become depleted. As a result, it would be greatly beneficial to be able to wirelessly transfer electrical energy to devices implanted within the body. While previous research has demonstrated the use of radio-frequency electromagnetic waves to supply power to implanted devices [11], concerns regarding associated factors such as electromagnetic coupling mean that the safe and reliable transfer of energy through the body is a continuing research goal [12].

The integration of a MEMS-based energy harvester with the implanted biomedical device may potentially address this challenge. In a typical implementation, an external source of energy could be used to mechanically excite a resonant MEMS structure, with the resulting motion being converted into electrical energy via integrated transducers. As demonstrated in [13], ultrasonic waves can be effectively used to transfer acoustic energy to a receiver implanted within soft tissue, and therefore represents an excitation method that is particularly suited to implanted biomedical applications (see Fig. 1). The transfer of energy via the use of ultrasonic waves has been demonstrated in [12], and has been shown to be an effective method of mechanically exciting an implanted microstructure [14].

An ultrasonically-excited MEMS energy harvester has been demonstrated in [15]-[17], where a 2-degree of freedom 
(DOF) device was fabricated using a silicon-on-insulator (SOI) MEMS process. The device featured a central proof mass which was designed to resonate in the in-plane directions when an external source of ultrasonic waves was directed at the device. Electrostatic transducers were implemented to generate electrical energy from the vibrations of the mass, with experimental testing showing that the harvester was able to successfully charge a load capacitor.

One common motivation for designing multi-DOF energy harvesters is to increase the effective harvesting bandwidth of the system. This can be done by designing individual DOFs to possess a slightly different resonance frequency, which allows the harvester to respond to a greater range of exciting vibration frequencies [18]. Alternatively, such multi-DOF harvesters can be used to harvest energy from vibrations sourced from different directions, rather than different frequencies. In a 1-DOF system, any misalignment with the direction of the exciting vibrations results in a significant reduction in power output, however the use of multiple harvesting DOFs in orthogonal directions greatly reduces this problem. For example, the 2-DOF system presented in [19] allows energy to be harvested from vibrations in any direction within the plane of the device.

\section{A NOVEl 3-DOF ENERGy HARVESTER}

This paper extends the work reported in [17] by presenting a novel 3-DOF MEMS energy harvester that is designed to use an external source of ultrasonic waves for mechanical excitation. The harvester features mechanical resonance modes in both the in-plane ( $\mathrm{x}$ and $\mathrm{y}$ ) directions as well as the out-of-plane $(\mathrm{z})$ direction, meaning that the device is able to harvest electrical energy regardless of its orientation with respect to the ultrasonic waves. This mechanism is of particular benefit when the orientation of the harvester cannot be guaranteed or controlled, as in an implanted biomedical application. The mechanical energy of the oscillating proof mass is converted into electrical energy via comb-finger electrostatic transducers, with individual harvesting mechanisms being employed for each of the harvester's degrees of freedom.

While a 3-DOF MEMS harvester was first demonstrated in [20], the device was fabricated using a nickel-based MEMS process that stipulated the use of relatively large feature sizes. The geometric properties of the chosen fabrication process limited the effectiveness of the device's electrostatic transducers, leading to a lower harvested power than demonstrated in [17]. In contrast, the device presented in this paper uses a conventional SOI process, allowing the full effectiveness of the integrated electrostatic transducers to be maintained.

\section{A. In-Plane Harvesting Mode}

The in-plane operation of the 3-DOF harvester is based on the principle of parallel kinematic design, and is an evolution of the 2-DOF harvesting mechanism presented in [17]. As shown in Fig. 2a, the device features a large central proof mass which is used to absorb incoming ultrasonic waves. A series of beam flexures are connected around the edges of the mass, which facilitate its displacement in the in-plane directions. These flexures effectively decouple the $\mathrm{x}$ and $\mathrm{y}$ motions of

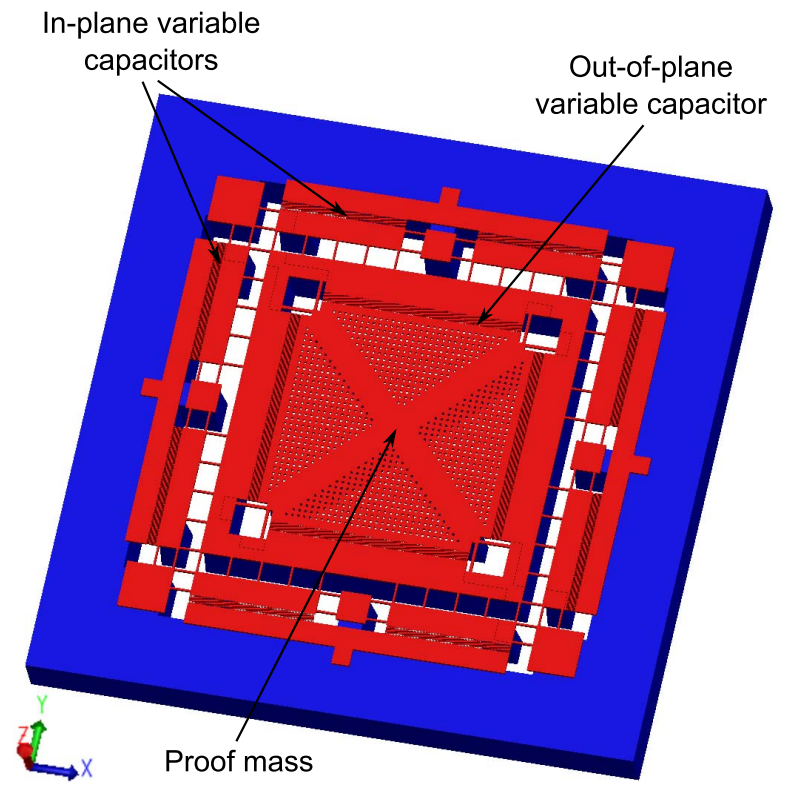

(a)

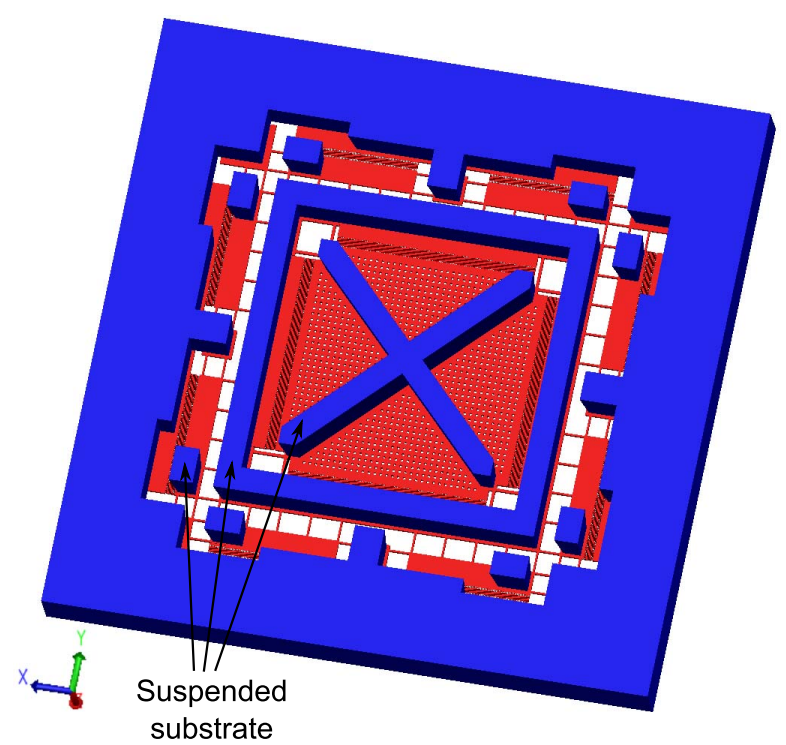

(b)

Fig. 2. a) Schematic diagram of 3-DOF ultrasonic energy harvester b) Reverse view showing suspended substrate structures.

the mass, and are designed such that the harvester's first inplane resonance modes are at the desired frequency.

To convert the in-plane displacements of the proof mass into harvested electrical power, electrostatic transducers are implemented in the form of in-plane, overlap-varying comb finger electrodes. These electrodes are positioned around the periphery of the harvester, with separate electrodes being used to harvest displacements along the $\mathrm{x}$ and $\mathrm{y}$ axes.

\section{B. Out-of-Plane Harvesting Mode}

In addition to the in-plane harvesting mechanism, the 3-DOF MEMS harvester features a mechanical resonance mode that is designed to allow ultrasonic waves to be harvested in the out-of-plane (z-axis) direction. This is achieved 


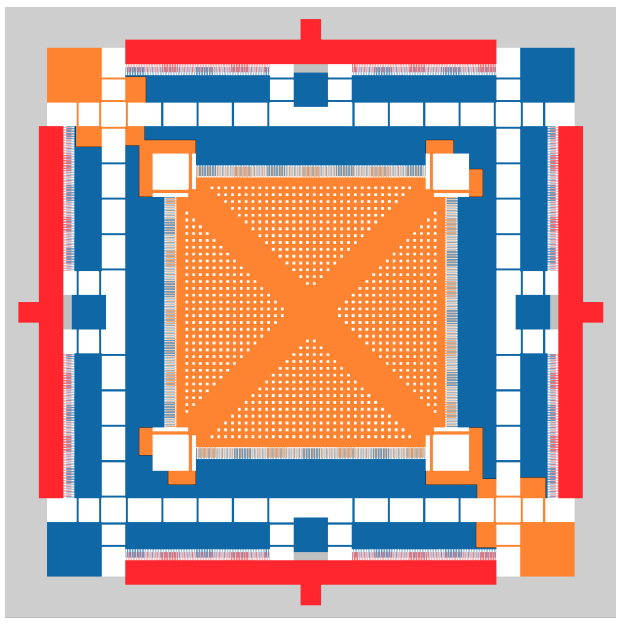

Fig. 3. MEMS harvester electrode layout. Different colors are electrically isolated due to etched areas in the SOI layer, with the substrate being used to mechanically link adjacent sections.

by subdividing the central proof mass to create a smaller, independent mass that is connected to the remainder of the movable structure via a secondary set of flexures. These flexures are designed such that the first resonance mode of this smaller mass is in the out-of-plane direction. This mechanical arrangement effectively means that the out-of-plane harvesting mechanism is nested serially within the in-plane harvesting structure. While the entire mass structure moves in unison in the in-plane directions, the out-of-plane resonance mode is based on relative displacement between the smaller nested mass and the surrounding frame.

An additional electrostatic transducer is used to facilitate energy harvesting using the z-axis displacement of the nested proof mass. In this case, a variable capacitor is implemented through the addition of comb finger electrodes to both the nested sub-mass and the surrounding structure. During the operation of the harvester's out-of-plane harvesting mechanism, the time-varying displacement of the nested mass leads to out-of-plane variations in the overlap between the comb fingers to provide the capacitance variations required for energy harvesting to take place.

Compared with the size of the entire device, the dimensions of the nested mass are relatively large (approximately $2 \mathrm{~mm} \times$ $2 \mathrm{~mm}$ ) in order to provide a large surface area for the capturing of incident ultrasonic waves. However, if the nested mass were comprised solely of the relatively thin silicon layer employed in a conventional SOI process, this structure would possess a very low mechanical stiffness in the out-of-plane direction. With a low thickness-to-width ratio, the silicon mass would tend to act like a membrane rather than a stiff mechanical structure, making it difficult to achieve a mechanical resonance mode at ultrasonic frequencies as desired.

To increase the out-of-plane stiffness of the nested mass, the substrate of the SOI process is utilized to mechanically reinforce the silicon layer. The substrate underneath the harvester is etched to leave a cross-shaped structure spanning the width of the nested silicon mass, which has the effect of allowing the silicon layer to maintain its rigidity when being displaced in the out-of-plane direction. This nested

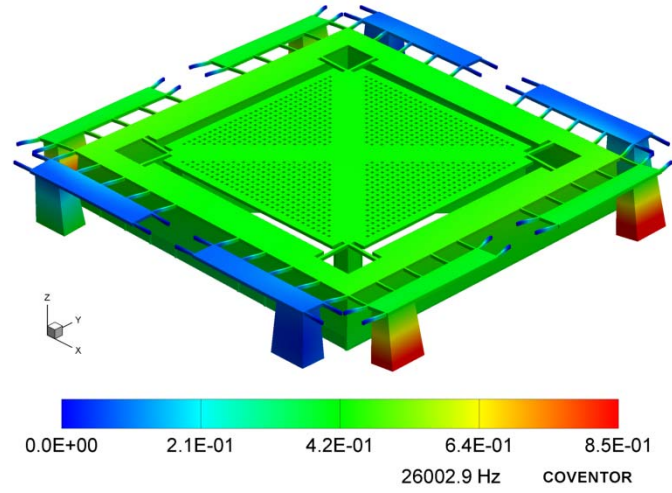

(a)
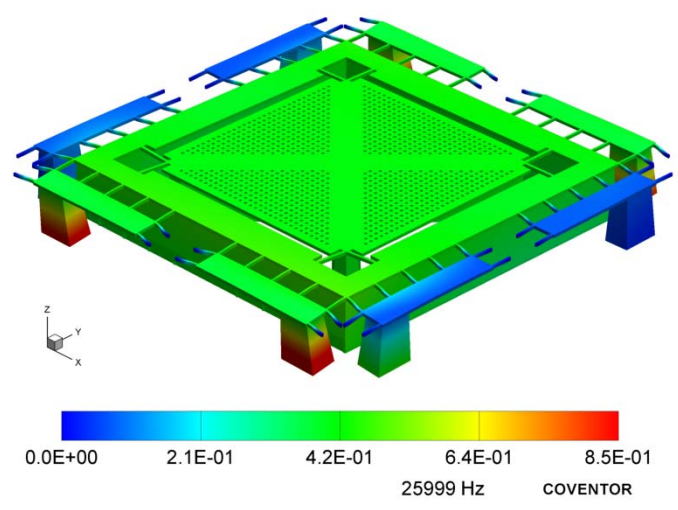

(b)

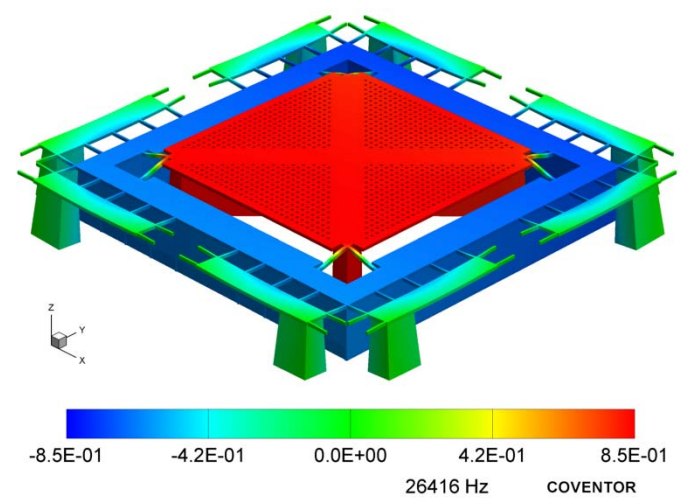

(c)

Fig. 4. Resonance modes simulated using CoventorWare. a) $\mathrm{X}$ mode. b) $\mathrm{Y}$ mode. c) $\mathrm{Z}$ mode.

mass is then connected to the remainder of the central mass structure via a secondary set of flexures, whose spring constant determines the z-axis resonance frequency of the out-of-plane mode. An additional ring of suspended substrate is attached to the outer section of the central mass structure to increase the mechanical stiffness of this section, enabling the desired relative $\mathrm{z}$-axis displacement between the nested mass and the surrounding structure in the out-of-plane resonance mode. These suspended substrate structures are shown in Fig. $2 b$.

As the 3-DOF system's out-of-plane harvesting mechanism is nested serially within the in-plane harvesting structure, the use of a conventional SOI process with a single conductive layer increases the complexity of routing an electrical con- 


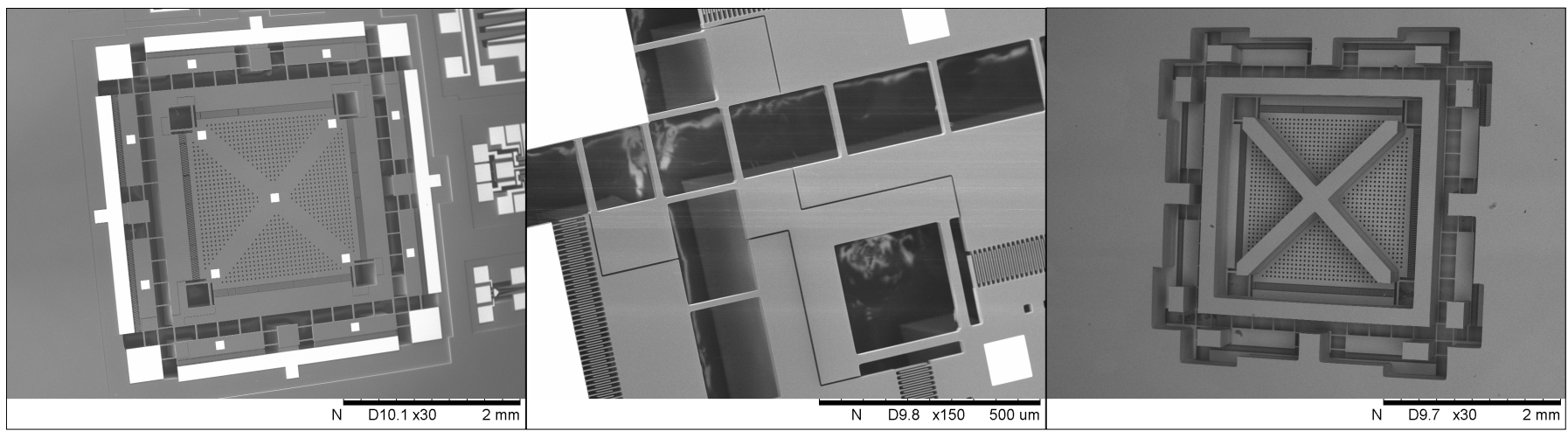

(a)

(b)

(c)

Fig. 5. a) SEM image of fabricated MEMS energy harvester. b) Close-up view showing cuts in SOI layer. c) Underside of fabricated harvester.

nection from the central harvesting electrode to the edge of the device for wirebonding. This is achieved in the design of the harvester through further utilization of the die's substrate, and by etching the main SOI layer in strategic locations to allow for electrical isolation of adjacent sections of the harvester. By allowing the substrate layer to remain in place underneath these areas, the electrically isolated components of the SOI layer remain mechanically connected through their attachment to the substrate via the buried oxide layer. This principle facilitates the creation of an electrical path from the nested mass at the center of the MEMS device to pads at the periphery of the design without compromising the operation of the in-plane harvesting mechanism. The electrically separated components of the MEMS harvester are shown in Fig. 3.

\section{Simulation of Resonance Modes}

In a typical application, the MEMS harvester would be mechanically excited by ultrasonic waves emitted by an external transmitter. To maximize the displacement of the harvester's mass structure, and consequently the power converted by the device, the frequencies of the harvester's primary resonance modes should be matched with the frequency of the ultrasonic excitation. As $25 \mathrm{kHz}$ is a common frequency for commercially available ultrasonic transmitters, this was chosen as the target frequency for the design of the harvester's resonance modes. Accordingly, the dimensions of the harvester's beam flexures and the etched area of the silicon layer were designed such that all three resonance modes $(\mathrm{x} / \mathrm{y} / \mathrm{z})$ are close to the desired frequency. A summary of the design parameters for the device is shown in Table I.

The resonance modes of the MEMS harvester were simulated using finite element analysis in the MEMS design software package CoventorWare. As shown in Fig. 4, each of the in-plane and out-of-plane modes are close to the targeted $25 \mathrm{kHz}$ resonance frequency.

\section{MEMS FABRICATION}

The 3-DOF MEMS harvester was fabricated via MEMSCAP's commercial SOIMUMPs prototyping process [21], with images of the fabricated device obtained via a scanning electron microscope (SEM) being shown in Fig. 5. The SOI
TABLE I

DESIGN PARAMETERS For 3-DOF MEMS ENERgy HARVESTER

\begin{tabular}{c|c}
\hline Parameter & Value \\
\hline \hline Comb finger gap & $3 \mu \mathrm{m}$ \\
\hline Comb finger width & $3 \mu \mathrm{m}$ \\
\hline Initial gap at end of in-plane comb finger & $20 \mu \mathrm{m}$ \\
\hline Flexure length $(\mathrm{X} / \mathrm{Y}$ axis mechanism) & $174 \mu \mathrm{m}$ \\
\hline Flexure width $(\mathrm{X} / \mathrm{Y}$ axis mechanism) & $15 \mu \mathrm{m}$ \\
\hline Flexure length $(\mathrm{Z}$ axis mechanism) & $260 \mu \mathrm{m}$ \\
\hline Flexure width $(\mathrm{Z}$ axis mechanism) & $23 \mu \mathrm{m}$ \\
\hline Proof mass dimensions $(\mathrm{X} / \mathrm{Y}$ axis mechanism) & $2.72 \mathrm{~mm} \times 2.72 \mathrm{~mm}$ \\
\hline Proof mass dimensions $(\mathrm{Z}$ axis mechanism) & $1.97 \mathrm{~mm} \times 1.97 \mathrm{~mm}$ \\
\hline Transducer rest capacitance $(\mathrm{X} / \mathrm{Y}$ axis) & $0.519 \mathrm{pF}$ \\
\hline Transducer rest capacitance $(\mathrm{Z}$ axis $)$ & $1.45 \mathrm{pF}$ \\
\hline
\end{tabular}

process features $25 \mu \mathrm{m}$ doped silicon for the main device layer containing the harvester's flexures, capacitive comb fingers, and main mass structures. Beneath the silicon layer is a $2 \mu \mathrm{m}$ thick buried oxide layer, which provides electrical isolation between adjacent silicon structures.

The MEMS die's substrate consists of $400 \mu$ m-thick silicon, which is largely removed from underneath the harvester via a deep reactive ion etch. However, as noted in Section II-B, a number of substrate structures are left in certain areas to create mechanical linkages and to increase the out-of-plane stiffness of the silicon layer. These suspended substrate elements are shown in Fig. 5c.

\section{EXPERIMENTAL CHARACTERIZATION}

\section{A. Mechanical Resonance Mode Identification}

The die containing the fabricated MEMS harvester was fixed to a custom PCB, which possesses a hole to enable the free movement of the device's suspended substrate structures. Electrical connections between the PCB and the harvester's electrical pads are made via gold bonding wires. An example of the experimental setup is shown in Fig. 6.

Initial testing of the fabricated harvester involved experimentally measuring the frequencies of the device's mechanical resonance modes. This was carried out using a 


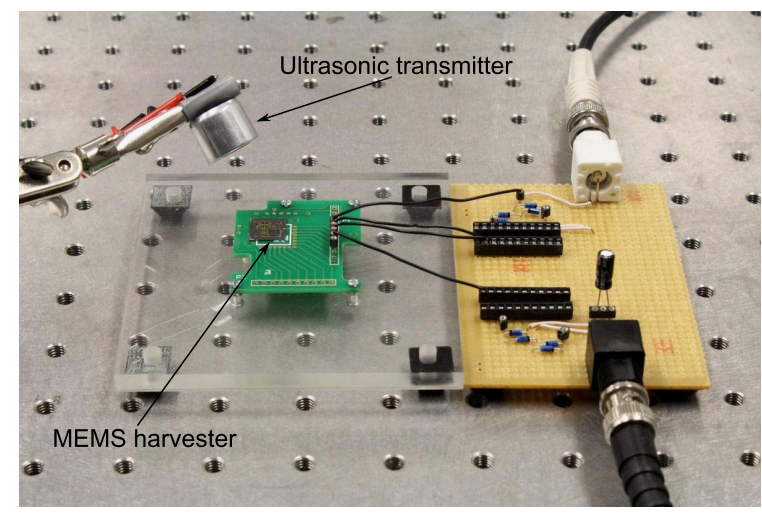

Fig. 6. Photo showing MEMS harvester experimental setup.
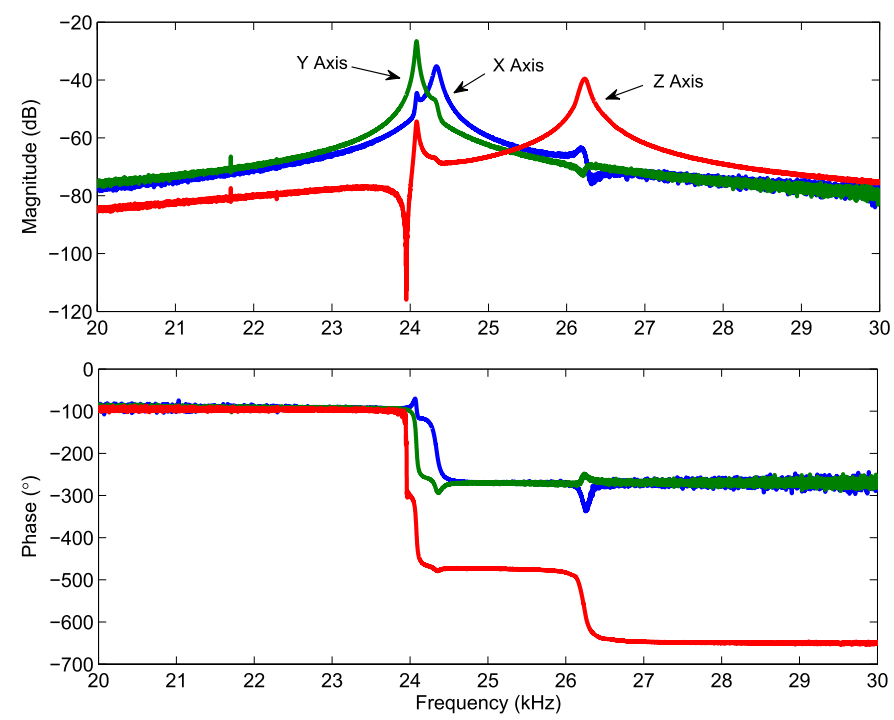

Fig. 7. Frequency response of fabricated harvester obtained using 3D vibrometer.

Polytec MSA-050-3D Micro System Analyzer (MSA), which features three independent laser Doppler vibrometers that directly measure the vibrations of a mechanical structure. These vibrations are resolved into their $\mathrm{x}-, \mathrm{y}-$, and $\mathrm{z}$-axis components, allowing each in-plane and out-of-plane mode to be individually identified.

To mechanically excite the resonance modes of the 3-DOF harvester while being analyzed with the MSA, a smallamplitude chirp signal superimposed on a $60 \mathrm{~V}$ DC bias was applied directly to the harvester's electrostatic transducers. While an external ultrasonic transmitter could equally have been used to excite the device, in this case the use of the harvester's electrostatic transducers as an actuating mechanism achieves the same result, and avoids the influence of a transmitter's dynamics on the resulting measurements. The frequency response measured by the MSA is shown in Fig. 7. As shown, there is a clear peak in the magnitude plot for each of the three orthogonal axes, indicating the locations of the designed resonance modes. These peaks are located at $24.30 \mathrm{kHz}, 24.08 \mathrm{kHz}$, and $26.23 \mathrm{kHz}$ for the $\mathrm{x}-, \mathrm{y}-$, and $\mathrm{z}$-axis modes respectively. These are close to the frequency of $25 \mathrm{kHz}$ targeted during the design of the harvester, however there are nevertheless some variations from the desired frequency in the fabricated device. The symmetry of the harvester design in the in-plane directions is reflected in the closeness of the $\mathrm{x}$ - and $\mathrm{y}$-axis modes, while the difference in frequency between these modes and the z-axis mode can be attributed to the use of a flexure mechanism that is independent of the in-plane mechanism. There is a strong correlation between the thickness of the main silicon layer and the outof-plane stiffness of the flexures used for the $\mathrm{z}$-axis harvesting mechanism, and therefore any slight deviation from the values used for the finite element simulation will result in a deviation from the simulated value in the fabricated device.

The MSA was also used to verify the shape and nature of the fabricated harvester's resonance modes. By scanning its vibrometers across the surface of an object, the MSA is able to generate a 3D representation of the vibrational modes generated by the mechanically excited device. From prior experience, it is known that the surface of the main silicon layer produced by the SOIMUMPs fabrication process does not sufficiently scatter the laser beams generated by the MSA's vibrometer to produce reliable measurements. For this reason, a thin layer of gold has been deposited in certain areas across the surface of the harvester's moving structures to provide points at which the vibrometer can be used to measure the device's vibrations. By combining the measurements taken by the vibrometer at the defined scan points, the mode shapes of the fabricated device were determined and are shown in Fig. 8. It can be seen from the figure that the mode shapes closely represent those obtained via the finite element simulations shown in Fig. 4, and only differ in frequency from the simulated values by a relatively small amount as previously discussed.

\section{B. Frequency Response Measurement Using Electrostatic Transducers}

The electrical output of the MEMS harvester's electrostatic transducers was used to verify the mechanical resonance modes identified using the MSA. The transducer for each of the harvester's three axes was connected to the input of a Stanford Research Systems SR780 signal analyzer, while a $60 \mathrm{~V} \mathrm{DC}$ voltage was applied to the transducers to provide the electrical bias required for the harvesting of energy from the variable capacitors. For practical use, a MEMS harvester's electrostatic transducers would be biased through methods such as the integration of electret materials as demonstrated in [22] and [23], or by using part of the harvested energy through the addition of external circuitry, as seen in [24] and [25]. In this case, however, the use of an external electrical source as the bias is sufficient for the purpose of characterizing this proof-of-concept device.

For this test, an off-the-shelf ultrasonic transmitter was utilized to provide the required mechanical excitation for the harvester. A Prowave 250SR160 transmitter was used, whose center frequency of approximately $25 \mathrm{kHz}$ is close to the frequencies of the harvester's main resonance modes in the in-plane and out-of-plane directions. The transmitter was placed close to the MEMS harvester at a $45^{\circ}$ angle to each of 


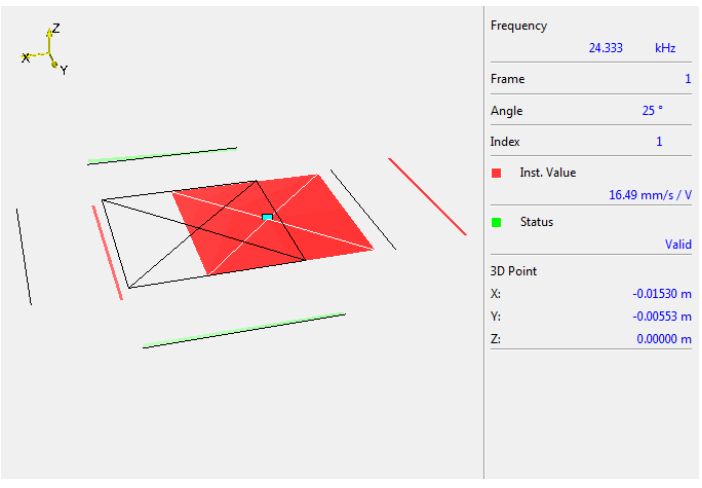

(a)

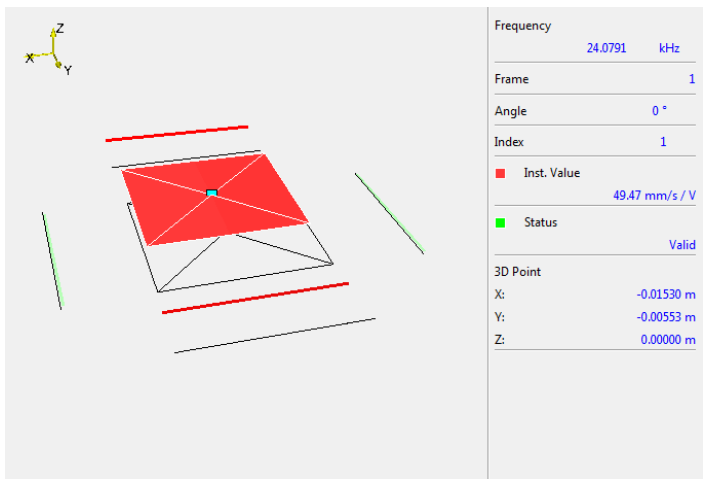

(b)

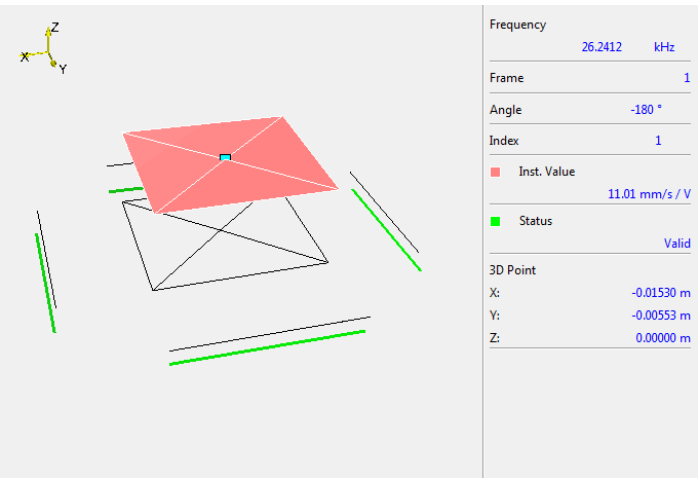

(c)

Fig. 8. Visualization of resonance modes experimentally identified using 3D vibrometer. a) $\mathrm{X}$ mode. b) $\mathrm{Y}$ mode. c) $\mathrm{Z}$ mode.

the device's three axes, and was driven by a $20 \mathrm{~V}_{\mathrm{rms}}$ wideband chirp signal. The resulting frequency response is shown in Fig. 9. The three resonance modes of the device are clearly evident in the signal analyzer's output, and are consistent with the resonance frequencies identified using the MSA. This test indicates that the previously-identified mechanical modes are successfully exploited through the use of the electrostatic transducers to convert the device's mechanical vibrations into electrical energy.

The main benefit of a MEMS harvester with three mechanical degrees of freedom is its ability to harvest power from the exciting ultrasonic transmitter regardless of the transmitter's orientation with respect to the harvester. The harvester's ability to respond to ultrasonic excitation from different directions was demonstrated by performing a series

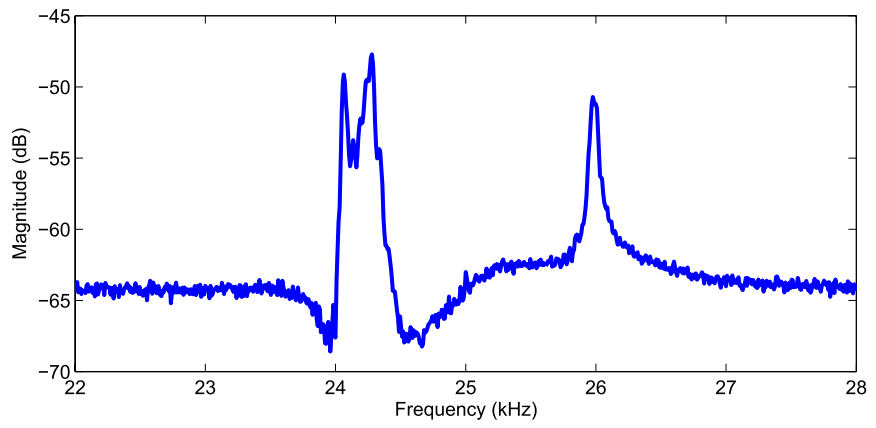

Fig. 9. Frequency response of MEMS harvester obtained using a signal analyzer, with excitation provided by an ultrasonic transmitter positioned at $45^{\circ}$ to all three axes.

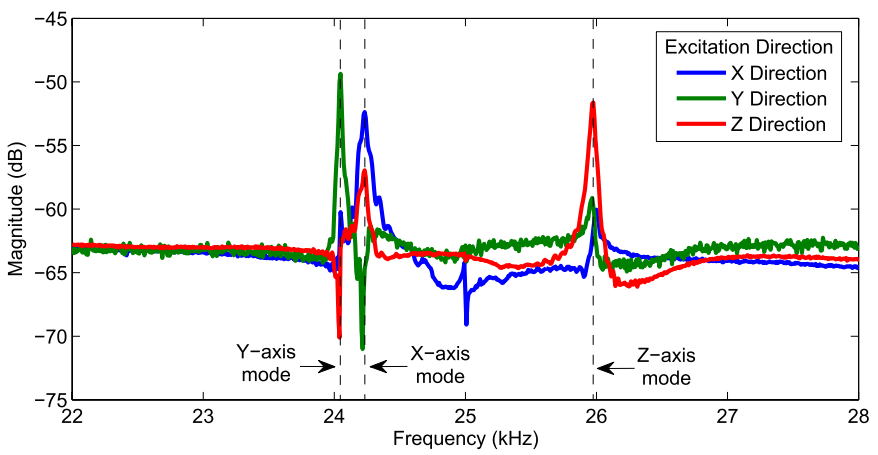

Fig. 10. Harvester frequency response measured using a signal analyzer. The responses from three separate tests are shown, with the ultrasonic transmitter being repositioned for each test to excite each axis individually.

of frequency response measurements using the signal analyzer, with the ultrasonic transmitter being aligned with one of the three main axes of the harvester during each test. The transmitter was again driven by a $20 \mathrm{~V}_{\text {rms }}$ chirp signal as in the previous test, while the transducers for all three axes were connected in parallel to the signal analyzer's input. The resulting measurements are shown in Fig. 10. It can be seen that for each excitation direction there is one dominant peak in the frequency response, and that the frequency of the dominant peak is different for each excitation direction. The frequencies of these peaks match those of the individual mechanical resonance modes previously identified in Fig. 7, verifying that the dominant vibrational mode is dependent on the alignment of the external transducer. This test therefore demonstrates the decoupled nature of the three designed resonance modes in the fabricated device. While for each test there are smaller peaks at the other resonance frequencies that indicate some excitation of the other modes, this can be attributed to both mechanical cross-coupling within the structure of the harvester and the effect of the ultrasonic transmitter's relatively wide beam angle of $85^{\circ}$.

\section{Measurement of Harvested Power}

Having determined the resonance frequencies of the fabricated device, its harvesting effectiveness was assessed by demonstrating the charging of a storage capacitor. The harvester's electrostatic transducers were each connected to a full-wave diode rectifier, with a $1 \mu \mathrm{F}$ low-ESR electrolytic 


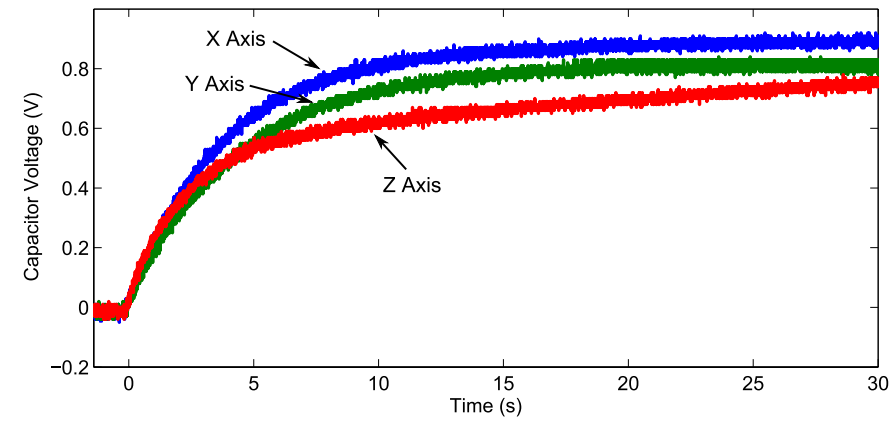

Fig. 11. Increasing voltage on storage capacitor due to harvesting from ultrasonic waves. Each of the three plots represents a separate test, showing the combined output of the harvester when the ultrasonic transmitter is aligned with one of the harvester's primary axes as denoted by the corresponding label.

capacitor being used to collect the harvested electrical energy. The time-varying voltage on the storage capacitor was measured using an oscilloscope connected via a Stanford SR560 low-noise voltage preamplifier with unity gain and an input impedance of $100 \mathrm{M} \Omega$. A $60 \mathrm{~V}$ electrical bias was again applied to each electrostatic transducer from an external power source.

For each test, the ultrasonic transmitter was placed approximately $5 \mathrm{~cm}$ from the MEMS die and directed along one of the harvester's main axes. The transmitter was driven by a $20 \mathrm{~V}_{\mathrm{rms}}$ sinusoidal signal at the frequency of the corresponding resonance mode, as identified via the previous frequency response measurements.

Fig. 11 shows the resulting increases in the voltage on the storage capacitor when the ultrasonic transmitter is activated at $t=0$. Each plot in the figure shows the total power output of the device when the transmitter is separately aligned with each of the primary axes of the harvester. The average power harvested by each axis of the device was determined via the method used in [17] to allow a meaningful comparison to be made with the earlier 2-DOF harvester. This involves calculating the increase in energy stored in the capacitor over the initial 15 second period, which is given by:

$$
P_{h}=\frac{1}{2 T} C V^{2}
$$

where $P_{h}$ is the average harvested power, $C$ is the capacitance of the storage capacitor, $V$ is the final voltage on the capacitor and $T$ is the time period over which the power is calculated. Based on these measurements, the electrical power harvested by the MEMS device was calculated to be $24.7 \mathrm{nW}, 19.8 \mathrm{nW}$, and $14.5 \mathrm{nW}$ for the $\mathrm{x}, \mathrm{y}$, and $\mathrm{z}$ axes respectively. These peraxis outputs are comparable with those obtained from the 2-DOF harvester in [17], however the omnidirectional harvester offers the significant advantage of allowing energy to be harvested from ultrasonic waves in all spatial directions.

Alternatively, it is proposed that a more meaningful representation of the harvester's output may be provided by calculating the generated instantaneous electrical power based on the initial rate of energy increase on the capacitor following the activation of the ultrasonic transmitter. Based on this method, the maximum power outputs were determined to
TABLE II

Estimated Transducer Capacitances Based on Measured DisPlaCEMENT OF HARVESTER MASS

\begin{tabular}{c|c|c}
\cline { 2 - 3 } & $C_{\min }$ & $C_{\max }$ \\
\hline $\mathrm{X}$ axis & $0.502 \mathrm{pF}$ & $0.536 \mathrm{pF}$ \\
\hline $\mathrm{Y}$ axis & $0.503 \mathrm{pF}$ & $0.536 \mathrm{pF}$ \\
\hline $\mathrm{Z}$ axis & $1.38 \mathrm{pF}$ & $1.45 \mathrm{pF}$ \\
\hline
\end{tabular}

be $49.4 \mathrm{nW}, 35.8 \mathrm{nW}$, and $35.0 \mathrm{nW}$ for the $\mathrm{x}, \mathrm{y}$, and $\mathrm{z}$ axes respectively.

As the energy produced by the harvester is rectified and stored on a capacitor at a usable voltage (approximately $0.6 \mathrm{~V}-0.9 \mathrm{~V})$ it is readily available for the powering of an external load. Alternatively, additional power management or conditioning circuitry may be implemented to suit specific applications [26].

\section{Conversion Efficiency}

As noted in [24], the conversion efficiency of an electrostatic energy converter such as the device presented in this paper can be assessed by comparing the actual power delivered by the harvester with the theoretical output of an optimal electrostatic transduction mechanism.

The electrical energy harvested by the device during one cycle of the harvester's mechanical oscillations is given by [27]:

$$
W_{\text {harvested }}=\frac{U_{0}^{2}}{2} C_{\max }\left(\frac{C_{\max }}{C_{\min }}-1\right)
$$

where $U_{0}$ is the bias voltage supplied to the electrostatic transducers, and $C_{\max }$ and $C_{\min }$ are the maximum and minimum capacitances of the transducers throughout the vibration cycle, respectively. To determine these corresponding transducer capacitances, the time-varying displacement of the proof mass was measured using the MSA while the device was excited by the ultrasonic transmitter as described in Section IV-C. The measured time-varying vibrations of the harvester were determined to be sinusoidal in nature, as expected, with amplitudes of $1.31 \mu \mathrm{m}, 1.28 \mu \mathrm{m}$, and $1.22 \mu \mathrm{m}$ for the $\mathrm{x}-, \mathrm{y}-$, and $\mathrm{z}$-axis resonance modes respectively.

From these measured displacements, the maximum and minimum capacitances of each of the harvester's electrostatic transducers were estimated based on the expression for the capacitance of in-plane overlap transducers [28]:

$$
C=\frac{N \varepsilon_{0} L z}{d}
$$

where $N$ is the number of comb fingers, $\varepsilon_{0}$ is the permittivity of free space, $L$ is the overlapping finger length, $z$ is the overlapping finger height, and $d$ is the gap between the fingers. The resulting estimated capacitances are shown in Table II.

These capacitances were used together with (2) to yield $W_{\text {harvested, the optimal energy harvested per cycle. The optimal }}$ harvested power, $P_{\text {harvested, }}$, is obtained using $P_{\text {harvested }}=$ $W_{\text {harvested }} f$, where $f$ is the vibration frequency of the proof mass. By expressing the actual power outputs measured in Section IV-C as a percentage of $P_{\text {harvested, the conversion }}$ efficiencies of the $\mathrm{x}, \mathrm{y}$, and $\mathrm{z}$ axis components of the MEMS 
harvester were calculated to be $1.58 \%, 1.30 \%$, and $0.42 \%$ respectively. While low, these efficiencies are comparable with other electrostatic harvesters in the literature [27], and indicate that there is significant scope for further improving the performance of the device. This may involve further optimization of the comb-finger transducers for the achieved mechanical displacements, and the use of more sophisticated power management circuitry. The potential use of a chargeconstrained conversion mode through the implementation of electret materials may also have some implications for the overall efficiency of the system due to the added need to convert the generated outputs, which are typically on the order of several hundred volts, to more usable voltages [29].

\section{CONCLUSION}

A novel 3-DOF MEMS energy harvester has been presented in this paper, with potentially suitable applications including the powering of implanted biomedical devices. The harvester is designed to use an external source of ultrasonic waves to mechanically excite a resonant mass structure, and integrated electrostatic transducers are used to harvest electrical energy from the resulting displacements. The device is designed to have three resonance modes in the $\mathrm{x}, \mathrm{y}$, and $\mathrm{z}$ directions, meaning that energy can be harvested regardless of the device's orientation relative to the exciting ultrasonic transmitter.

The harvester is fabricated using a commercial SOI MEMS process, with experimental characterization showing that the device's resonance modes are close to the designed frequency of $25 \mathrm{kHz}$. Through the charging of a load capacitor, the $\mathrm{x}-, \mathrm{y}-$, and $\mathrm{z}$-axis harvesting mechanisms of the device were determined to generate $24.7 \mathrm{nW}, 19.8 \mathrm{nW}$, and $14.5 \mathrm{nW}$ of electrical power respectively from ultrasonic waves generated by an external transmitter.

The 3-DOF MEMS harvester successfully demonstrates the energy harvesting concept using a mechanism that is potentially suited to biomedical applications. Future work aims to address the additional challenges that must be met to create a practical realization of the system, including the efficient transfer of ultrasonic waves from human tissue into the harvester.

\section{REFERENCES}

[1] C. B. Williams and R. B. Yates, "Analysis of a micro-electric generator for microsystems," in Proc. 8th Int. Conf. Solid-State Sens. Actuators, Eurosens. IX (Transducers), Stockholm, Sweden, Jun. 1995 pp. $369-372$.

[2] S. Roundy, P. K. Wright, and J. Rabaey, "A study of low level vibrations as a power source for wireless sensor nodes," Comput. Commun., vol. 26, no. 11, pp. 1131-1144, Jul. 2003.

[3] P. D. Mitcheson, E. M. Yeatman, G. K. Rao, A. S. Holmes, and T. C. Green, "Energy harvesting from human and machine motion for wireless electronic devices," Proc. IEEE, vol. 96, no. 9, pp. 1457-1486, Sep. 2008.

[4] E. O. Torres and G. A. Rincón-Mora, "Electrostatic energy harvester and Li-ion charger circuit for micro-scale applications," in Proc. 49th IEEE Int. Midwest Symp. Circuits Syst., San Juan, Puerto Rico, USA, Aug. 2006, pp. 65-69.

[5] A. Harb, "Energy harvesting: State-of-the-art," Renew. Energy, vol. 36, no. 10, pp. 2641-2654, Oct. 2011.

[6] P. D. Mitcheson, T. C. Green, E. M. Yeatman, and A. S. Holmes, "Architectures for vibration-driven micropower generators," J. Microelectromech. Syst., vol. 13, no. 3, pp. 429-440, Jun. 2004.
[7] S. P. Beeby, M. J. Tudor, and N. M. White, "Energy harvesting vibration sources for microsystems applications," Meas. Sci. Technol., vol. 17, no. 12 , pp. $175-195$, Dec. 2006.

[8] P. C.-P. Chao, "Energy harvesting electronics for vibratory devices in self-powered sensors," IEEE Sensors J., vol. 11, no. 12, pp. 3106-3121, Dec. 2011.

[9] S. D. Nguyen, E. Halvorsen, and G. U. Jensen, "Wideband MEMS energy harvester driven by colored noise," J. Microelectromech. Syst., vol. 22, no. 4, pp. 892-900, Aug. 2013.

[10] M. R. Mhetre, N. S. Nagdeo, and H. K. Abhyankar, "Micro energy harvesting for biomedical applications: A review," in Proc. 3rd ICECT, Kanyakumari, India, Apr. 2011, pp. 1-5.

[11] M. D. Zimmerman, N. Chaimanonart, and D. J. Young, "In vivo RF powering for advanced biological research," in Proc. 28th IEEE EMBS Аnnu. Int. Conf., New York, NY, USA, Aug. 2006, pp. 2506-2509.

[12] S. Arra, J. Leskinen, J. Heikkila, and J. Vanhala, "Ultrasonic power and data link for wireless implantable applications," in Proc. 2nd Int. Symp. Wireless Pervas. Comput., San Juan, Puerto Rico, USA, Feb. 2007, pp. 567-571.

[13] S. Ozeri, D. Shmilovitz, S. Singer, and C.-C. Wang, "Ultrasonic transcutaneous energy transfer using a continuous wave $650 \mathrm{kHz}$ Gaussian shaded transmitter," Ultrasonics, vol. 50, no. 7, pp. 666-674, Jun. 2010.

[14] A. Denisov and E. Yeatman, "Stepwise microactuators powered by ultrasonic transfer," in Proc. Eng. Eurosens. XXV, Athens, Greece, Sep. 2011, pp. 685-688.

[15] Y. Zhu, S. O. R. Moheimani, and M. R. Yuce, "A 2-DOF wideband electrostatic transducer for energy harvesting and implantable applications," in Proc. IEEE Sensors, Oct. 2009, pp. $1542-1545$

[16] Y. Zhu, S. O. R. Moheimani, and M. R. Yuce, "Ultrasonic energy transmission and conversion using a 2-D MEMS resonator," IEEE Electron Device Lett., vol. 31, no. 4, pp. 374-376, Apr. 2010.

[17] Y. Zhu, S. O. R. Moheimani, and M. R. Yuce, "A 2-DOF MEMS ultrasonic energy harvester," IEEE Sensors J., vol. 11, no. 1, pp. 155-161, Jan. 2011

[18] G. D. Pasquale and A. Somà, "Low-frequency MEMS energy harvesters for the supplying of vehicle diagnostic systems," in Proc. Symp. DTIP MEMS/MOEMS, Seville, Spain, May 2010, pp. 134-139.

[19] U. Bartsch, J. Gaspar, and O. Paul, "Low-frequency two-dimensional resonators for vibrational micro energy harvesting," J. Micromech Microeng., vol. 20, no. 3, p. 035016, Mar. 2010.

[20] A. G. Fowler, S. O. R. Moheimani, and S. Behrens, "A 3-DOF MEMS ultrasonic energy harvester," in Proc. IEEE Sensors, Taipei, Taiwan, Oct. 2012, pp. 1-4.

[21] A. Cowen, G. Hames, D. Monk, S. Wilcenski, and B. Hardy. (2011). SOIMUMPs design handbook. MEMSCAP, Durham, NC, USA [Online]. Available: http://www.memscap.com

[22] U. Bartsch, J. Gaspar, and O. Paul, "A 2D electret-based resonant micro energy harvester," in Proc. IEEE 22nd Int. Conf. Micro Electro Mech. Syst., Sorrento, Italy, Jan. 2009, pp. 1043-1046.

[23] T. Sterken, P. Fiorini, K. Baert, R. Puers, and G. Borghs, "An electretbased electrostatic $\mu$-generator," in Proc. 12th Int. Conf. Solid-State Sens., Actuators, Microsyst. (Transducers), vol. 2. Boston, MA, USA, Jun. 2003, pp. 1291-1294.

[24] B. C. Yen and J. H. Lang, "A variable-capacitance vibration-to-electric energy harvester," IEEE Trans. Circuits Syst. I, Reg. Papers, vol. 53, no. 2, pp. 288-295, Feb. 2006.

[25] E. O. Torres and G. A. Rincón-Mora, "Self-tuning electrostatic energyharvester IC," IEEE Trans. Circuits Syst. II, Exp. Briefs, vol. 57, no. 10 , pp. 808-812, Oct. 2010.

[26] E. O. Torres and G. A. Rincon-Mora, "Electrostatic energy-harvesting and battery-charging CMOS system prototype," IEEE Trans. Circuits Syst. I, Reg. Papers, vol. 56, no. 9, pp. 1938-1948, Sep. 2009.

[27] P. Basset, D. Galayko, A. M. Paracha, F. Marty, A. Dudka, and T. Bourouina, "A batch-fabricated and electret-free silicon electrostatic vibration energy harvester," J. Micromech. Microeng., vol. 19, no. 11, p. 115025 , Nov. 2009.

[28] S. Roundy, P. K. Wright, and K. S. J. Pister, "Micro-electrostatic vibration-to-electricity converters," in Proc. ASME Int. Mech. Eng. Congr. Exposit., New Orleans, LA, USA, Nov. 2002, pp. 487-496.

[29] P. D. Mitcheson, T. C. Green, and E. M. Yeatman, "Power processing circuits for electromagnetic, electrostatic and piezoelectric inertial energy scavengers," Microsyst. Technol., vol. 13, nos. 11-12, pp. 1629-1635, Jul. 2007. 


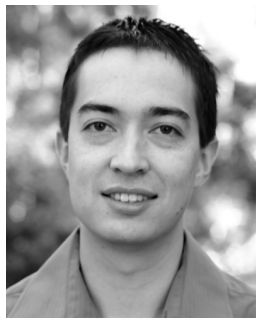

Anthony G. Fowler (S'10) was born in Taree, Australia. He received the Bachelor's degree in electrical engineering from the University of Newcastle, Callaghan, Australia, in 2010. He is the recipient of an Australian Postgraduate Award, and is currently pursuing the $\mathrm{Ph} . \mathrm{D}$. degree in electrical engineering at the same university.

His research interests include the design and analysis of novel MEMS devices for energy harvesting and nanopositioning applications.

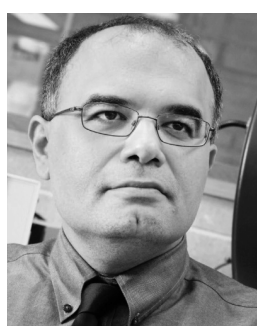

S. O. R. Moheimani (M'96, SM'00, F'11) received the Doctoral degree from the University of New South Wales at the Australian Defence Force Academy in Canberra, Australia, in 1996.

He joined the University of Newcastle in 1997 embarking on a new research program addressing the dynamics and control design issues related to highprecision mechatronic systems. He is the Founder and Director of the Laboratory for Dynamics and Control of Nanosystems, a multimillion-dollar stateof-the-art research facility. He has published over 300 refereed papers and five books and edited volumes. His current research interests include ultra-high-precision mechatronic systems, with particular emphasis on dynamics and control at the nanometer scale, including applications of control and estimation in nanopositioning systems for high-speed scanning probe microscopy, modeling and control of microcantilever-based devices, control of micro-actuators in microelectromechanical systems, and design, modeling and control of micro-machined nanopositioners for on-chip atomic force microscopy.

Professor Moheimani is a fellow of IEEE, IFAC and the Institute of Physics, U.K. His work has been recognized with a number of awards, including the IFAC Nathaniel B. Nichols Medal in 2014, the IFAC Mechatronic Systems Award in 2013, the IEEE Control Systems Technology Award in 2009, the Australian Research Council Future Fellowship in 2009, the IEEE Transactions on Control Systems Technology Outstanding Paper Award in 2007, the Australian Research Council Post Doctoral Fellowship in 1999, and several best student paper awards in various conferences. He has served on the editorial boards of a number of journals, including the IEEE/ASME TRANSACTIONS ON MECHATRONICS, the IEEE TRANSACTIONS ON CONTROL Systems Technology, and Control Engineering Practice. He currently chairs the IFAC Technical Committee on Mechatronic Systems, and has chaired several international conferences and workshops.

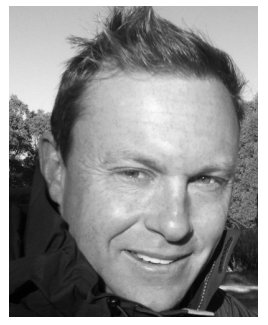

Sam Behrens (M'03) received B.Eng. (Mech.) and M.Eng. (Elec.) degrees from the University of Newcastle, NSW, Australia, in 1999 and 2001 respectively. Also in 2001, he received an Australian Postgraduate Award that allowed him to complete his Ph.D. in 2005 at the University of Newcastle. As a former member of the Laboratory for Dynamics and Control of Nanosystems, his Ph.D. in vibration control earned him a CSIRO OCE Postdoctoral Research Fellowship in vibration energy harvesting.

Dr. Behrens is presently based at CSIRO's Energy Centre as Demand Side Energy Systems capability/project leader. He oversees a multi-disciplinary research group consisting of 35 scientist/engineers, software developers and applied mathematicians. Key focuses of the group are integration of renewable energy into smart grids, smart grid control and novel power generation technologies. His personal research interests include wave energy harvesting, energy storage, power electronics, mechatronics systems and control of sound and vibration. He is the co-author of two book chapters, several patent applications and more than 55 journal and conference papers.

Dr Behrens' international reputation was recognized in 2009, when he was selected as part of a delegation of ten accomplished professionals from across Australia to partake in the Australian Korean Young Leader Exchange Program. He has been invited to present his work at a number of key energy harvesting international conferences, and he is an active reviewer for IOP Smart Materials and Structures, ASME Journal of Sound and Vibration and associate editor for IEEE/ASME International Conference on Advanced Intelligent Mechatronics. 